\title{
Erosão dentária em paciente jovem por influência dietética: relato de caso
}

\author{
Dental erosion in a young patient due to dietary influence: case report \\ Erosión dental en paciente joven debido a la influencia de la dieta: reporte de caso
}

Tamires Passadori Martins

ORCID: https://orcid.org/0000-0003-4153-1548

Universidade Estadual Paulista, Brasil

E-mail: tamires.passadori@unesp.br

Mayra Fernanda Ferreira

ORCID: https://orcid.org/0000-0003-0442-3126

Universidade Estadual Paulista, Brasil

E-mail: mayra.fernanda@unesp.br

Isabela Maria Passarela Gomes

ORCID: https://orcid.org/0000-0002-8734-4556

Universidade Estadual Paulista, Brasil

E-mail: isabela.m.gomes@unesp.br

Mayra Frasson Paiva

ORCID: https://orcid.org/0000-0002-4677-1420

Universidade Estadual Paulista, Brasil

E-mail: mfpaiva@gmail.com

Marcos Rogério de Mendonça

ORCID: https://orcid.org/0000-0001-8081-9144

Universidade Estadual Paulista, Brasil

E-mail: rogerio.mendonca@ unesp.br

Juliano Pelim Pessan

ORCID: https://orcid.org/0000-0002-1550-3933 Universidade Estadual Paulista, Brasil E-mail: juliano.pessan@unesp.br

Igor Renan Zen

ORCID: https://orcid.org/0000-0002-2524-4365 Universidade Estadual Paulista, Brasil E-mail: igor.zen@unesp.br

\begin{abstract}
Resumo
A erosão dentária é um processo crônico, caracterizado pela dissolução química da superfície dental, em decorrência da ação de ácidos de origem intrínseca, extrínseca ou idiopática. Estima-se que esta condição afete, mundialmente, cerca de $30-50 \%$ da dentição decídua e $20-45 \%$ da permanente. O objetivo deste trabalho foi relatar um caso clínico de erosão dentária moderada associada à alta ingestão de alimentos ácidos e fermantados. Paciente do sexo masculino, leucodermo, 8 anos, compareceu à Clínica de Odontopediatria da Faculdade de Odontologia de Araçatuba para consulta preventiva. Durante a anamnese, constatou-se consumo de refrigerante 2 vezes por semana, suco de frutas ácidas 2 vezes ao dia e bebida láctea açucarada $1 \mathrm{vez}$ ao dia. Ao exame clínico, foi observado ausência de lesões cariosas, tecido gengival sadio, entretanto, havia a presença de fluorose generalizada e desgaste dental erosivo generalizado, com características como brilho excessivo, lisura e lesões do tipo "cupping" na superfície oclusal dos molares decíduos, além de desgaste nas bordas incisais de incisivos e caninos. A conduta clínica imediata envolveu orientação de dieta e higiene aos responsáveis, enfatizando os fatores causais e a característica irreversível do desgaste dental erosivo. Foram realizadas quatro sessões de aplicação tópica de verniz fluoretado. O acompanhamento odontológico infantil e o diagnóstico precoce de desgastes dentários são de suma importância, a fim de minimizar os danos estruturais aos dentes decíduos e também prevenir danos à dentição permanente.
\end{abstract}

Palavras-chave: Erosão dentária; Desgaste dos dentes; Dieta; Odontopediatria.

\begin{abstract}
Dental erosion is a chronic process characterized by chemical dissolution of the tooth surface due to the action of acids of intrinsic extrinsic or idiopathic origin. It is estimated that this condition affects about $30-50 \%$ of primary dentition and $20-45 \%$ of permanent teeth worldwide. This work aimed to report a clinical case of moderate dental erosion associated with a high intake of acidic and fermented foods. Male patient, Caucasian, 8 years old, attended the Pediatric Dentistry Clinic of Araçatuba Dental School for preventive consultation. During the anamnesis, there was consumption of soft drinks twice a week, acidic fruit juice twice a day, and a sugary milk drink once a day. On
\end{abstract}


clinical examination, the absence of carious lesions, healthy gingival tissue were observed. However, there was the presence of generalized fluorosis and generalized erosive tooth wear, with characteristics such as excessive shine, smoothness, and cupping-type lesions on the occlusal surface of the primary molars, in addition, to wear on the incisor edges of incisors and canines. The immediate clinical approach involved guidance on diet and hygiene to those responsible, emphasizing the causal factors and the irreversible character of erosive tooth wear. Four sessions of topical application of fluoride varnish were carried out. Child dental care and early diagnosis of tooth wear are of paramount importance to minimize structural damage to primary teeth and prevent damage to permanent dentition.

Keywords: Tooth erosion; Tooth wear; Diet; Pediatric dentistry.

\section{Resumen}

La erosión dental es un proceso crónico, caracterizado por la disolución química de la superficie del diente, debido a la acción de ácidos de origen intrínseco, extrínseco o idiopático. Se estima que esta condición afecta, en todo el mundo, alrededor del 30-50\% de la dentición temporal y del 20-45\% de los dientes permanentes. El objetivo de este trabajo fue reportar un caso clínico de erosión dental moderada asociada a una alta ingesta de alimentos ácidos y fermentados. Paciente de sexo masculino, caucásico, de 8 años, que acudió a la Clínica de Odontopediatría de la Facultad de Odontología de Araçatuba para consulta preventiva. Durante la anamnesis, hubo consumo de refrescos dos veces por semana, jugo de frutas ácidas dos veces al día y una bebida láctea azucarada una vez al día. Al examen clínico se observó ausencia de lesiones cariosas, se observó tejido gingival sano, sin embargo, hubo presencia de fluorosis generalizada y desgaste dental erosivo generalizado, con características como excesivo brillo, tersura y lesiones tipo ahuecamiento en la superficie oclusal de los molares temporales, además del desgaste en los bordes incisivos de incisivos y caninos El abordaje clínico inmediato implicó la orientación en la dieta e higiene a los responsables, enfatizando los factores causales y la característica irreversible del desgaste dental erosivo. Se realizaron cuatro sesiones de aplicación tópica de barniz fluorado. La monitorización dental infantil y el diagnóstico precoz del desgaste de los dientes son de suma importancia para minimizar el daño estructural a los dientes temporales y también prevenir daños en la dentición permanente.

Palabras clave: Erosión de los dientes; Desgaste de los dientes; Dieta; Odontología pediátrica.

\section{Introdução}

A erosão dentária caracteriza-se por um processo químico, sem envolvimento bacteriano, que resulta na dissolução da estrutura dental devido à exposição crônica a ácidos (Schlueter et al., 2020). Estes ácidos podem ser de origem intrínseca ou extrínsica. São considerados agentes intrínsecos aqueles relacionados à doenças psicossomáticas que provocam regurgitação do ácido gástrico ou diminuição do fluxo salivar, enquanto dentre os extrínsecos estão dieta, meio ambiente e medicamentos (Lussi et al., 2004; Lussi \& Jaeggi, 2008; Barbour \& Lussi, 2014; Jordão et al., 2020). O processo de erosão dentária ainda sofre influência de fatores como pH salivar, morfologia da estrutura dentária e posicionamento dos dentes em relação os tecidos moles bucais e língua (Zero \& Lussi, 2005; Hara et al., 2006).

Nas últimas décadas houve um aumento na prevalência de casos de erosão dentária, sob influência do estilo de vida moderno e maior consumo de bebidas ácidas proporcionadas pelo mesmo (Jaeggi \& Lussi, 2014; Al-Dlaigan et al., 2017; Carvalho \& Lussi, 2020). A dentição decídua apresenta maior vulnerabilidade ao desgaste dental erosivo, uma vez que os dentes possuem esmalte menos mineralizado e, consequentemente, com menor microdureza e maior solubilidade quando comparados à dentição permanente (Carvalho et al., 2014; Assunção et al., 2019). Além disso, estima-se que esta condição afete, mundialmente, 30-50\% dos dentes decíduos e 20-45\% da dentição permanente, e que 30\% dos adolescentes possuam erosão dentária (Salas et al., 2015; Murakami et al., 2016; Chan et al., 2020). Com relação às características clínicas, dentes acometidos pela erosão dentária apresentam superfícies lisas, brilhantes e vítreas. Também é possível identificar lesões côncavas em que a dentina é visível, sendo estas denominadas “cupping” (Lussi \& Jaeggi, 2008). Com sua progressão, o quadro de erosão dentária pode levar à hipersensibilidade dentinária, perda da dimensão vertical de oclusão e prejuízos estéticos, o que contribui diretamente para uma menor qualidade de vida do paciente (Li \& Bernabé, 2016).

A utilização de produtos fluoretados, especialmente os de alta concentração, está entre os principais meios de tratamento e prevenção do desgaste dental erosivo (Lussi et al., 2019). Estes produtos atuam como protetores através da 
formação de fluoreto de cálcio $\left(\mathrm{CaF}_{2}\right)$, formando uma barreira física à penetração de ácidos e um reservatório de íons a serem liberados durante os desafios erosivos (Lussi et al., 2019). Entretanto, o pH dos agentes erosivos é menor que o da fluorapatita e hidroxiapatita, fazendo com que o flúor tenha uma ação limitada sobre a prevenção do processo erosivo (Assis et al., 2011; Magalhães et al., 2011). Portanto, faz-se necessário aumentar os números de aplicações dos produtos ou associar o fluoreto a outros compostos, como titânio e estanho, a fim de potencializar seu efeito protetor (Lussi et al., 2019).

Diante do exposto, o presente trabalho tem como objetivo relatar um caso clínico de erosão dentária em paciente pediátrico.

\section{Metodologia}

O presente estudo é um relato de caso clínico, descritivo e qualitativo. Esta modalidade de estudo particulariza-se pela abordagem de um assunto específico, estudando-o de maneira minuciosa (Pereira et al., 2018). Em relação aos aspectos éticos e morais, informações quanto ao prognóstico, riscos e benefícios foram fornecidas aos responsáveis legais pelo paciente através de um Termo de Consentimento Livre e Esclarecido (TCLE), posteriormente assinado pelos mesmos.

\section{Caso Clínico}

Paciente do sexo masculino, leucodermo, oito anos de idade, compareceu à Clínica de Odontopediatria da Faculdade de Odontologia de Araçatuba (FOA-UNESP) para consulta preventiva. Os responsáveis relataram, durante anamnese, que o paciente apresentava bom estado de saúde geral. Ao exame clínico, foi observado ausência de lesões cariosas, tecido gengival sadio e a presença de fluorose em dentição permanente (Figura 1).

Figura 1. Vista vestibular dos incisivos permanentes superiores e inferiores com fluorose dentária.

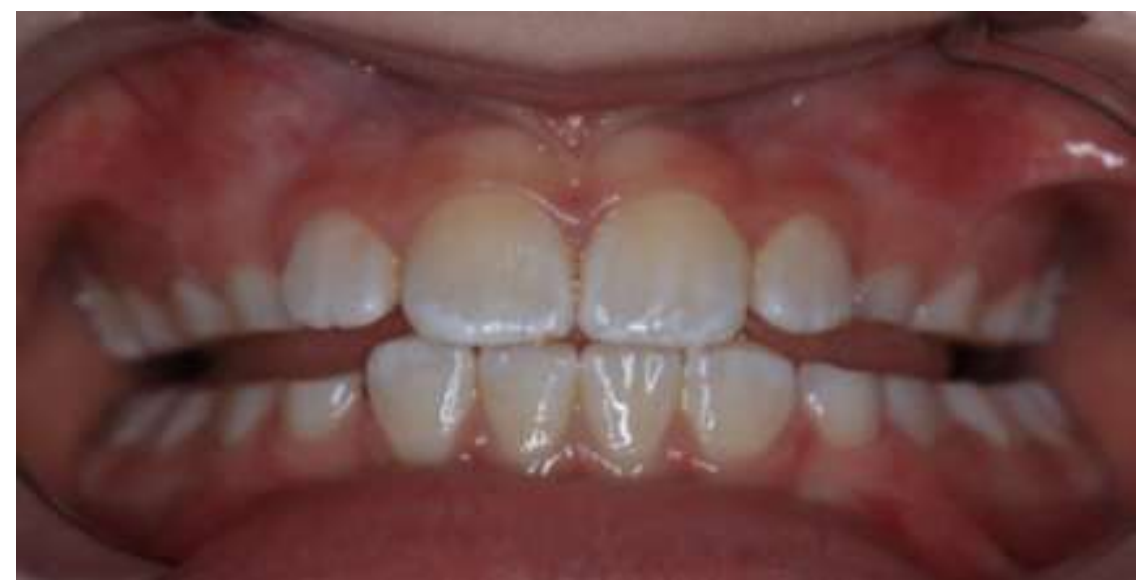

Fonte: Autores.

Observou-se, também, a presença de desgaste dental erosivo generalizado. Características como brilho excessivo, lisura e lesões do tipo “cupping” foram identificadas nas superfícies oclusais de molares e caninos decíduos, além de desgastes iniciais em bordas incisais e superfícies oclusais de incisivos e primeiros molares permanentes, respectivamente. 
Research, Society and Development, v. 10, n. 15, e85101522624, 2021

(CC BY 4.0) | ISSN 2525-3409 | DOI: http://dx.doi.org/10.33448/rsd-v10i15.22624

Figura 2. Desgaste dental erosivo generalizado (a), com lesões tipo "cupping" (b) e acometimento inicial em molares permanentes (c).

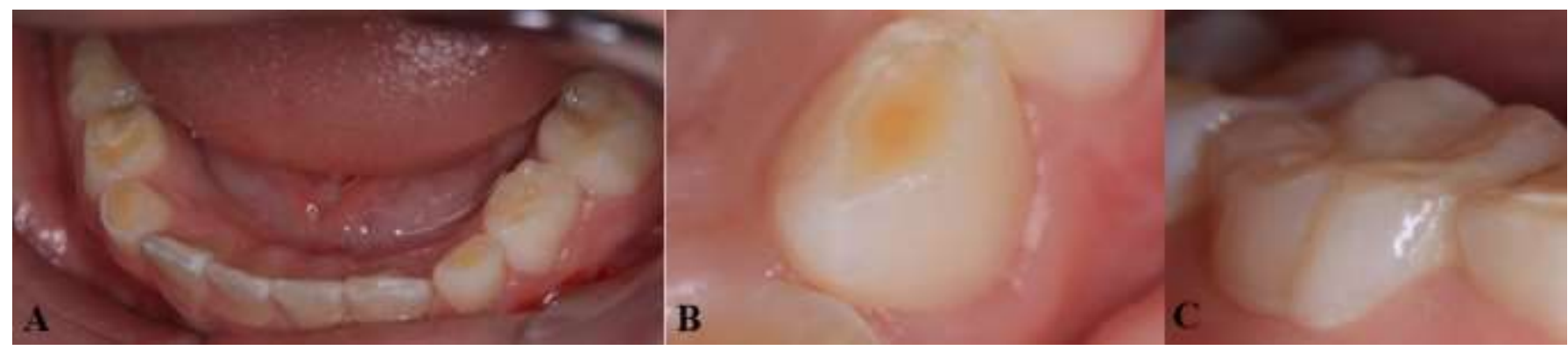

Fonte: Autores.

Considerando o quadro de desgaste dental erosivo generalizado, utilizou-se o índice de diagnóstico "Basic Erosive Wear Examination - BEWE" (Exame Básico de Desgaste Erosivo), que classificou o paciente como "médio risco", sugerindo avaliação da dieta e higiene oral, fluoretação e acompanhamento da condição. Desta forma, solicitou-se a realização de um diário alimentar para analisar características específicas da dieta da criança. O paciente ingeria sucos cítricos duas vezes ao dia, bebida láctea açucarada no mínimo uma vez ao dia e consumia refrigerante ao menos duas vezes na semana. Esta alta ingestão de bebidas ácidas foi considerada o agente causal do quadro de erosão dentária. O tratamento consistiu em quatro sessões de aplicações de verniz fluoretado Duraphat ${ }^{\circledR}$ (Colgate, Alemanha) com o auxílio de microbrush (Figura 3), aliadas à orientação da dieta e higienização bucal, enfatizando a necessidade de mudança de hábitos, visto a característica irreversível do desgaste dental erosivo.

Figura 3. Aplicação de verniz fluoretado Duraphat ${ }^{\circledR}$ (Colgate, Alemanha).

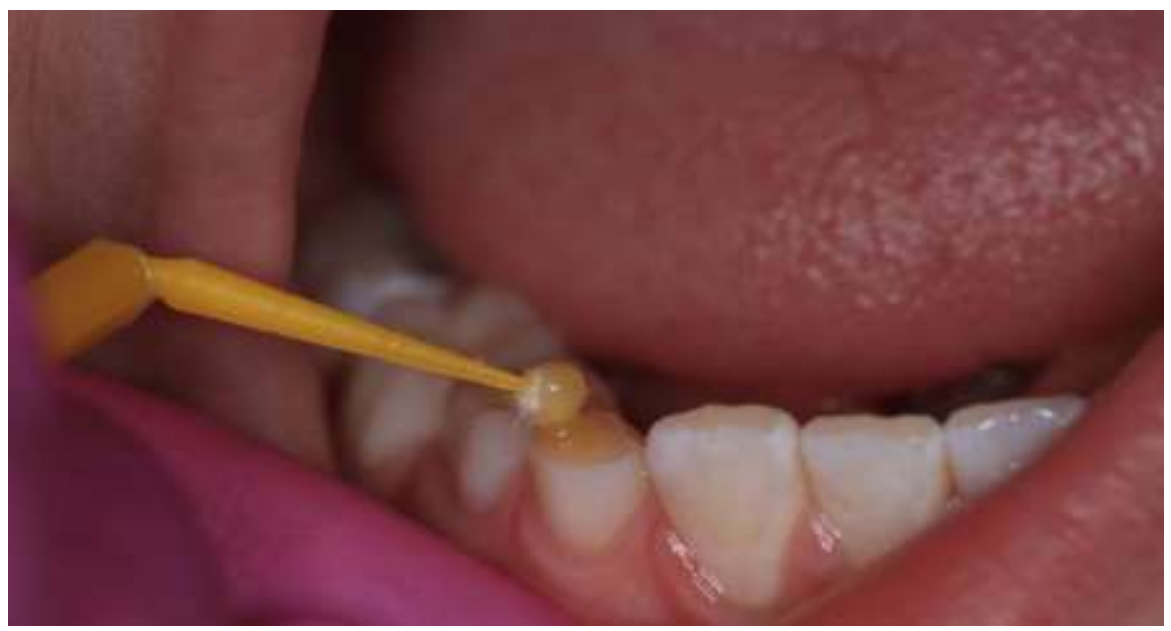

Fonte: Autores.

\section{Discussão}

A erosão dentária é uma condição irreversível e cumulativa, frequentemente associada a outros tipos de desgastes, como abrasão (Lussi \& Jaeggi, 2008; Salas et al., 2015). Nos últimos anos, notou-se um aumento na prevalência de erosão dentária em crianças e adolescentes, sendo o estilo de vida moderno e os hábitos alimentares proporcionados pelo mesmo um dos principais contribuintes para este crescimento (Barbour \& Lussi, 2014; Salas et al., 2015; Murakami et al., 2016; Chan et 
al., 2020; Maltarollo et al., 2020). Estima-se que esta condição afete cerca de 30-50\% dos dentes decíduos e 20-45\% da dentição permanente, mundialmente (Murakami et al., 2016; Chan et al., 2020).

O diagnóstico inadequado ou tardio, bem como a falta de intervenções preventivas, leva à perda avançada da estrutura dentária, comprometendo vitalidade, função e estética do dente (Salas et al., 2015; Li \& Bernabé, 2016). Características como superfícies oclusais e incisais lisas, brilhantes e com aspecto vítreo, assim como lesões tipo “cupping”, são aspectos comuns em dentes acometidos pelo desgaste dental erosivo (Lussi \& Jaeggi, 2008). Com relação à classificação do quadro de erosão dentária, o mesmo pode ser considerado leve, moderado ou severo, bem como localizado ou generalizado, de acordo com o nível de perda da estrutura dentária e o número de dentes acometidos, respectivamente (Bartllet et al., 2008; Schlueter et al., 2020). Dentes com desgaste moderado ou severo podem envolver exposição dentinária (Schlueter et al., 2020). No caso clínico exposto, o paciente apresentava lesões com as mesmas características que as descritas na literatura, sendo um quadro de desgaste dental erosivo generalizado com níveis de acometimento dentário entre leve e moderado, sendo classificado como "médio risco" de acordo com o índice BEWE.

Dentre as formas de prevenção e tratamento da erosão dentária, encontram-se a utilização de produtos fluoretados e a eliminação e controle de fatores etiológicos (Lussi et al., 2019; Carvalho \& Lussi, 2020; Jordão et al., 2020). Os produtos fluoretados atuam por meio da formação de $\mathrm{CaF}_{2}$, que age como barreira física e reservatório de íons, dificultando a penetração de ácidos e liberando íons durante desafios erosivos, respectivamente (Lussi et al., 2019). A utilização de produtos com maiores concentrações de flúor e em maior número de aplicações promovem maior proteção ao desgate dental erosivo (Magalhães et al., 2011). O tratamento instiuído no presente caso envolveu quatro sessões de aplicação de verniz fluoretado, o qual possui alta concentração de íons flúor. Entretanto, apenas a utilização de produtos fluoretados não é capaz de impedir o processo de erosão dentária, sendo necessárias medidas para eliminação dos fatores etiológicos e, por vezes, um tratamento multidisciplinar (Magalhães et al., 2011; Barbour \& Lussi, 2014; Carvalho \& Lussi, 2020; Jordão et al., 2020).

Os hábitos alimentares têm apresentado forte influência sobre o desenvolvimento de quadros de erosão dentária em crianças e adolescentes, sendo o consumo de bebidas ácidas, especialmente sucos cítricos e refrigerantes, um dos principais fatores extrínsecos (Barbour \& Lussi, 2014; Carvalho \& Lussi, 2020; Chan et al., 2020). Estas bebidas apresentam valores médios de potencial hidrogeniônico $(\mathrm{pH})$ entre 2.5-3.4, os quais são mais baixos que aqueles considerados críticos para a hidroxiapatita e fluorapatita (Assis et al., 2011). O consumo de refrigerantes e sucos cítricos aumenta em 20\% o desenvolvimento de erosão dentária (Salas et al., 2015). Desta forma, faz-se necessário um acompanhamento da dieta do paciente, através de diário alimentar, a fim de avaliar o potencial erosivo de todas as bebidas e alimentos ingeridos pelo paciente, uma vez que, a partir dessas informações, será possível elaborar estratégias preventivas e de tratamento para minimizar a progressão ou impedir o desenvolvimento do desgaste dental erosivo (Carvalho \& Lussi, 2020). Através da solicitação de um diário alimentar, identificou-se que o paciente em questão ingeria sucos cítricos duas vezes ao dia, bem como bebida láctea fermentada, fatores que contribuiam para o desenvolvimento do desgaste dental erosivo, por este motivo, os pais receberam instrruções de dieta, a fim de previnir a progressão do quadro de erosão dentária.

\section{Considerações Finais}

Diante do exposto, ressalta-se a importância do diagnóstico precoce da erosão dentária em consonância com a compreensão e eliminação dos fatores etiológicos, a fim de minimizar danos à dentição decídua e previnir o acometimento de dentes permanentes. O desgate dentário causado pelo quadro é irreversível e gera perda da dimensão vertical, hipersensibilidade dentinária e prejuízos estéticos. Portanto, consultas regulares ao Odontopediatra são imprescindíveis para prevenção, diagnóstico precoce e tratamento adequado. 
Research, Society and Development, v. 10, n. 15, e85101522624, 2021

(CC BY 4.0) | ISSN 2525-3409 | DOI: http://dx.doi.org/10.33448/rsd-v10i15.22624

\section{Referências}

Assis, C. D., Barin, C. S., \& Ellensohn, R. M. (2011). Estudo do potencial de erosão dentária de bebidas ácidas. Journal of Health Sciences, 13(1).

Assunção, C. M., Schlueter, N., Rodrigues, J. A., Carvalho, T. S., \& Lussi, A. (2019). Do fluoride toothpastes have similar preventive effect in permanent and primary teeth against erosive tooth wear? International journal of paediatric dentistry, 29(2), 228-236.

Al-Dlaigan, Y. H., Al-Meedania, L. A., \& Anil, S. (2017). The influence of frequently consumed beverages and snacks on dental erosion among preschool children in Saudi Arabia. Nutrition journal, 16(1), 1-6.

Barbour, M. E., \& Lussi, A. (2014). Erosion in relation to nutrition and the environment. Erosive Tooth Wear, 25, $143-154$.

Bartlett, D., Ganss, C., \& Lussi, A. (2008). Basic Erosive Wear Examination (BEWE): a new scoring system for scientific and clinical needs. Clinical oral investigations, 12(1), 65-68.

Carvalho, T. S., Lussi, A., Jaeggi, T., \& Gambon, D. L. (2014). Erosive tooth wear in children. Erosive tooth wear, $25,262-278$.

Carvalho, T. S., \& Lussi, A. (2020). Acidic beverages and foods associated with dental erosion and erosive tooth wear. The Impact of Nutrition and Diet on Oral Health, 28, 91-98.

Chan, A. S., Tran, T. T. K., Hsu, Y. H., Liu, S. Y. S., \& Kroon, J. (2020). A systematic review of dietary acids and habits on dental erosion in adolescents. International journal of paediatric dentistry, 30(6), 713-733.

Hara, A. T., Lussi, A., \& Zero, D. T. (2006). Biological factors. Dental Erosion, 20, 88-99.

Jaeggi, T., \& Lussi, A. (2014). Prevalence, incidence and distribution of erosion. Erosive Tooth Wear, 25, 55-73.

Jordão, H. W., Coleman, H. G., Kunzmann, A. T., \& McKenna, G. (2020). The association between erosive toothwear and gastro-oesophageal reflux-related symptoms and disease: A systematic review and meta-analysis. Journal of dentistry, 95, 103284.

Li, M. H., \& Bernabé, E. (2016). Tooth wear and quality of life among adults in the United Kingdom. Journal of dentistry, 55, 48-53.

Lussi, A., Jaeggi, T., \& Zero, D. (2004). The role of diet in the aetiology of dental erosion. Caries research, 38(Suppl. 1), 34-44.

Lussi, A., \& Jäeggi, T. (2008). Erosion—diagnosis and risk factors. Clinical oral investigations, 12(1), 5-13.

Lussi, A., Buzalaf, M. A. R., Duangthip, D., Anttonen, V., Ganss, C., João-Souza, S. H., ... \& Carvalho, T. S. (2019). The use of fluoride for the prevention of dental erosion and erosive tooth wear in children and adolescents. European archives of paediatric dentistry, 20(6), $517-527$.

Magalhães, A. C., Wiegand, A., Rios, D., Buzalaf, M. A. R., \& Lussi, A. (2011). Fluoride in dental erosion. Fluoride and the oral environment, 22, 158-170.

Maltarollo, T. H., Pedron, I. G., Medeiros, J. M. F., Kubo, H., Martins, J. L., \& Shitsuka, C. (2020). A erosão dentária é um problema!. Research, Society and Development, 9(3), e168932723-e168932723.

Murakami, C., Tello, G., Abanto, J., Oliveira, L. B., Bonini, G. C., \& Bönecker, M. (2016). Trends in the prevalence of erosive tooth wear in Brazilian preschool children. International journal of paediatric dentistry, 26(1), 60-65.

Pereira, A. S., Shitsuka, D. M., Parreira, F. J., \& Shitsuka, R. (2018). Metodologia da pesquisa científica.

Salas, M. M. S., Nascimento, G. G., Huysmans, M. C., \& Demarco, F. F. (2015). Estimated prevalence of erosive tooth wear in permanent teeth of children and adolescents: an epidemiological systematic review and meta-regression analysis. Journal of Dentistry, 43(1), 42-50.

Schlueter, N., Amaechi, B. T., Bartlett, D., Buzalaf, M. A. R., Carvalho, T. S., Ganss, C., ... \& Lippert, F. (2020). Terminology of erosive tooth wear: consensus report of a workshop organized by the ORCA and the Cariology Research Group of the IADR. Caries research, 54(1), 2-6.

Zero, D. T., \& Lussi, A. (2005). Erosion—chemical and biological factors of importance to the dental practitioner. International dental journal, 55, 285-290. 